\title{
Desenvolvimento do milho e fertilidade do solo após aplicação de lodo de curtume e fosforita
}

\author{
Fabio F. de Araújo ${ }^{1}$, Carlos S. Tiritan ${ }^{1}$, Helder M. Pereira ${ }^{1} \& 0$ svanir Caetano Júnior ${ }^{1}$
}

\begin{abstract}
RESU M O
Avaliar as alterações químicas no solo e o crescimento de plantas de milho em um Argissolo Vermelho Amarelo distroférrico, em dois cultivos sucessivos após a aplicação de lodo de curtume e fosfato natural, foi o que norteou este trabaIho. Estudou-se, em condições de casa de vegetação, o efeito de duas doses de lodo (2,5 e 5,0 Mg ha-1) in natura e compostado associado ao fosfato natural, sobre 0 crescimento e concentrações de nutrientes no milho e a fertilidade do solo. Verificou-se efeito significativo das doses de lodo in natura sobre o desenvolvimento do milho quando comparado com os tratamentos N PK e testemunha. A concentração de fósforo disponível no solo foi incrementada após a aplicação de lodo de curtume. 0 lodo, no entanto, não supriu a quantidade de potássio necessária para o desenvolvimento das plantas no segundo cultivo, mas o lodo de curtume "in natura" promoveu maior acúmulo de $\mathrm{N}$ e P na planta e melhor desenvolvimento do milho no primeiro cultivo, o que não se repetiu no segundo cultivo; o lodo de curtume compostado apresentou maior acúmulo de $\mathrm{N}$ no segundo cultivo, representando também maior estabilização do $\mathrm{N}$ no resíduo compostado. A associação do lodo de curtume com a fosforita proporcionou ganhos no desenvolvimento do milho e aumento do P disponível no solo.
\end{abstract}

Palavras-chave: resíduo industrial, reciclagem, compostagem, nutrição de plantas

\section{Development of maize and soil fertility after application of tannery sludge and rock phosphate}

\begin{abstract}
This study was elaborated with the objective of evaluating the chemical alterations in soil and the growth of maize in Red Yellow distrophic soil, in two successive crops, after tannery sludge application. Under greenhouse conditions, the effects were studied of two doses of sludge (2.5 e $5.0 \mathrm{Mg} \mathrm{ha}^{-1}$ ) "in natura" and associated to phosphate rock, on the growth and nutrition of maize and soil fertility. Significant effects were observed for the sludge dose "in natura" compared with N PK treatment and control. The addition of sludge in the soil increased the indicators of soil fertility. These treatments showed maximum values of soluble phosphorus in the soil. The tannery sludge failed to supply the amount of $\mathrm{K}$ for the development of the plants in the second crop. It was observed that the tannery sludge "in natura" increased the concentration of $\mathrm{N}$ and $\mathrm{P}$ in the plant, resulting in good development of maize in the first crop. The composted tannery sludge presented a high concentration of $\mathrm{N}$ in the second crop. This confirmed stabilization of nitrogen in the composting. The association of residue with rock phosphate increased the corn development with increase of available P in the soil.
\end{abstract}

Key words: industrial residue, composting, recycling, plant nutrition 


\section{INTRODUÇÃO}

Dentre os nutrientes presentes nos fertilizantes, o fósforo se constitui em um dos mais limitantes para a cultura do milho, especialmente em solos tropicais que, em geral, apresentam baixo teor de fósforo disponível e reação ácida, cujas características, aliadas às altas taxas de adsorção do fósforo, têm sido consideradas as limitações mais severas para a utilização desses solos e para o aumento da produtividade, já que se fazem necessárias aplicações de altas doses de fósforo (Lopes, 1984). Ressalta-se, ainda, que os fosfatos são recursos naturais não renováveis, escassos e sem sucedâneos devendo, portanto, ter utilização eficaz.

A utilização de fosfato solúvel em água constitui a forma mais satisfatória de suprir a necessidade das plantas porém há necessidade de adubações, além das quantidades requeridas pelas plantas, em virtude das perdas para o solo (Tsunechiro \& Ferreira, 1996). A fosforita (fosfato natural) é uma rocha fosfatada com aproximadamente $24 \%$ de fósforo total, de baixo custo e com reduzido teor de fósforo solúvel; este produto pode constituir-se em excelente fonte de fósforo de baixo custo para os agricultores, desde que atenda às necessidades de fósforo nos cultivos anuais. A disponibilidade de fósforo solúvel pela aplicação de fosfatos naturais está sujeita a lenta solubilização do fosfato, o que limita sua aplicação em culturas de rápido crescimento (Kucey et al., 1989). A associação de fosfatos reativos com resíduos orgânicos, enriquecidos com microrganismos solubilizadores de fósforo, tem proporcionado resultados satisfatórios na solubilização do fósforo (Hussain et al., 2001). Por outro lado, o simples fato da presença de matéria orgânica disponibiliza fósforo em fosfatos insolúveis provavelmente pela atividade microbiana saprofítica (Branco et al., 2001).

Nos últimos anos a atividade agrícola tem sofrido profundas modificações em razão do aumento do custo de produção com reflexos na rentabilidade da cultura, especialmente em grãos como o milho. A adubação é reconhecidamente o fator que mais afeta a produtividade e a sustentabilidade da atividade, de modo que, no País, o consumo de adubo pela cultura do milho tem crescido acentuadamente nos últimos anos, em função do aumento por unidade de área.

A destinação do lodo de curtume para a agricultura é uma alternativa que merece destaque, desde que convenientemente aplicada. Atualmente, com as novas tecnologias de reciclagem do cromo na indústria, este resíduo tem apresentado concentrações baixas deste metal, o que poderá reduzir o impacto ambiental da referida técnica. Vários trabalhos comprovam a eficiência de resíduos de curtume como bons fertilizantes e corretivos de acidez dos solos (Konrad \& Castilhos, 2002; Ferreira et al., 2003; Araújo et al., 2005); neste sentido, Castilhos (1998) verificou que na aplicação de lodo mais fósforo e potássio, os rendimentos foram semelhantes aos do tratamento calcário mais NPK e cerca de 16 vezes superiores ao rendimento da testemunha. Por sua vez, a adição de resíduos de curtume pode elevar a concentração de sais no solo, sobretudo o lodo de caleiro, rico em cálcio e sódio. Em trabalho de aplicação de doses crescentes de lodo de curtume na cultura do milho, observou-se aumento de produção de matéria seca da cultura (Araújo et al., 2005); contudo, os autores citam que o monitoramento dos teores de sais e cromo deve ser constante em razão do risco de contaminação do solo. O monitoramento do solo é um dos itens exigidos pela Companhia de Tecnologia em Saneamento Ambiental de São Paulo - CETESB, quando da apresentação de projetos de aplicação de lodo de curtume na agricultura. Considerando a inexistência de trabalhos de associação de lodo de curtume ao fosfato natural e a necessidade de se aliar o interesse agronômico ao ambiental buscou-se, com este trabalho, avaliar os efeitos da associação do lodo de curtume "in natura" e compostado, com fosfato natural sobre componentes de produção de milho, alteração da fertilidade do solo e acúmulo de cromo no solo.

\section{MATERIAL E MÉTODOS}

O lodo de curtume utilizado foi produzido no decantador primário (reunião de todos os efluentes gerados no processo), do curtume Vitapelli, localizado no município de Presidente Prudente o qual, após desaguamento, apresentava consistência pastosa. O curtume faz a precipitação e reciclo do cromo, proporcionando o resíduo final com baixa concentração de cromo. Os resultados da análise química do lodo estão expressos na Tabela 1.

Tabela 1. Composição química (\%) no lodo de curtume utilizado no experimento

\begin{tabular}{lcccccccccc}
\hline Lodo & Umidade & $\mathbf{N}$ & $\mathbf{P}$ & $\mathbf{K}$ & $\mathbf{C a}$ & $\mathbf{M g}$ & $\mathbf{S O}_{4}$ & $\mathbf{C r}$ & $\mathbf{F e}$ & $\mathbf{M n}$ \\
\hline Natural & 80,10 & 2,91 & 0,61 & 0,1 & 12,8 & 1,08 & 1,30 & 0,18 & 0,27 & 0,51 \\
Compostado & 10,32 & 1,02 & 0,55 & 0,12 & 4,85 & 0,38 & 0,53 & 0,07 & 0,57 & 0,71 \\
\hline
\end{tabular}

Para obtenção de composto a partir do lodo de curtume, realizou-se compostagem prévia e, como resíduo estruturante, bagaço de cana, na proporção de duas partes de bagaço para uma parte de lodo. A compostagem foi conduzida em leiras durante 90 dias e realizados reviramentos mecânicos semanais; a análise química do composto final obtido está expressa na Tabela 1.

O solo (argissolo vermelho amarelo distroférrico) (EMBRAPA, 1999), utilizado como substrato para a condução do experimento, representa um solo típico da região de Presidente Prudente e foi submetido a análise química de fertilidade antes da instalação do experimento, sendo encontrado os seguintes valores: $\mathrm{pH}\left(\mathrm{CaCl}_{2}\right)-5,8$; mat. orgânica (g $\left.\mathrm{dm}^{3}\right)-10,0 ; \mathrm{Ca}\left(\mathrm{mmol}_{\mathrm{c}} \mathrm{dm}^{3}\right)-9,0 ; \mathrm{Mg}\left(\mathrm{mmol}_{\mathrm{c}} \mathrm{dm}^{3}\right)-6,0 ; \mathrm{K}$ $\left(\mathrm{mmol}_{\mathrm{c}} \mathrm{dm}^{3}\right)-2,2 ; \mathrm{P}\left(\mathrm{mg} \mathrm{dm}^{3}\right)-30,0 ; \mathrm{S}\left(\mathrm{mg} \mathrm{dm}^{3}\right)-1,3$; CTC $\left(\mathrm{mmol}_{\mathrm{c}} \mathrm{dm}^{3}\right)-42,0 ; \mathrm{V}(\%)-40,0 . \mathrm{O}$ experimento conduzido com o cultivo do milho (variedade Agromen AGL25023) foi realizado em condições de casa-de-vegetação do Centro de Ciências Agrárias da Universidade do Oeste Paulista, em Presidente Prudente, SP. Utilizaram-se vasos plásticos com capacidade para $10 \mathrm{~kg}$ de solo e após a efetivação dos tratamentos foram realizados dois cultivos sucessivos, o primeiro iniciado em 09/09/2005 e o segundo, sem adição de lodo e fertilizantes, em 12/12/2005, ambos com duração de 60 dias 
com os mesmos vasos. A umidade dos vasos foi mantida na capacidade de campo, por meio de pesagens diárias, de modo a repor as perdas por evapotranspiração.

Usou-se, como fonte de fósforo, o fosfato natural (Fosforita alvorada) que apresenta a concentração de $24 \%$ de $\mathrm{P}_{2} \mathrm{O}_{5}$ total e $4 \%$ de fósforo solúvel em água. O fosfato foi adicionado ao solo na ocasião do plantio do milho.

Empregaram-se, para estabelecimento dos tratamentos e como referência, as taxas de aplicação de 2,5 a $5,0 \mathrm{Mg}$ $\mathrm{ha}^{-1}$ quantidades próximas das utilizadas em projetos de aplicação de lodo de curtume em áreas agrícolas da região. Esses tratamentos, considerando-se a mineralização de $35 \%$ do $\mathrm{N}$ total do lodo (Tabela 1 ), fornecerão valores próximos de 15 a $50 \mathrm{~kg}$ de $\mathrm{N}$ para a cultura. A forma de aplicação do lodo e composto foi superficial, ou seja, os resíduos foram distribuídos na superfície do solo acondicionado nos vasos $72 \mathrm{~h}$ antes do plantio do milho. Todos os tratamentos foram associados à aplicação de 100 $\mathrm{kg}$ de $\mathrm{P}_{2} \mathrm{O}_{5}$. No tratamento NPK as fontes de $\mathrm{N}$ foi a uréia, de $\mathrm{P}$ foi o superfosofato simples e de $\mathrm{K}$ foi o cloreto de potássio. Os tratamentos ficaram, então, assim constituídos:

$\mathrm{T}_{1}$ - Testemunha (Solo nas condições naturais)

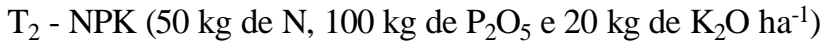
$\mathrm{T}_{3}$ - NK (50 kg de N e $20 \mathrm{~kg} \mathrm{de} \mathrm{K}_{2} \mathrm{O} \mathrm{ha}^{-1}$ ) + Fosforita $\left(100 \mathrm{~kg}\right.$ de $\mathrm{P}_{2} \mathrm{O}_{5}$ ha $\left.^{-1}\right)$

$\mathrm{T}_{4}$ - Lodo $\left(2,5 \mathrm{Mg} \mathrm{ha}^{-1}\right)+$ Fosforita $\left(100 \mathrm{~kg} \mathrm{ha}^{-1} \mathrm{de}_{2} \mathrm{O}_{5}\right)$

$\mathrm{T}_{5}$ - Lodo $2 \mathrm{x}\left(5,0 \mathrm{Mg} \mathrm{ha}^{-1}\right)+$ Fosforita $\left(100 \mathrm{~kg} \mathrm{ha}^{-1} \mathrm{de}_{2} \mathrm{O}_{5}\right)$

$\mathrm{T}_{6}$ - Composto $\left(2,5 \mathrm{Mg} \mathrm{ha}^{-1}\right)+$ Fosforita $\left(100 \mathrm{~kg} \mathrm{ha}^{-1} \mathrm{de}_{2} \mathrm{O}_{5}\right)$

$\mathrm{T}_{7}$ - Composto $2 \mathrm{x}\left(5,0 \mathrm{Mg} \mathrm{ha}^{-1}\right)+$ Fosforita $\left(100 \mathrm{~kg} \mathrm{ha}^{-1}\right.$ de $\mathrm{P}_{2} \mathrm{O}_{5}$ )

O crescimento do milho foi avaliado pela produção de matéria seca e altura de plantas. Para determinação da altura das plantas efetuou-se medição, com auxílio de fita métrica, do comprimento correspondente à base do solo até a ponta da última folha, antes da coleta das plantas; para determinação de matéria seca o material foi secado em estufa de ventilação forçada, sob temperatura de 75 ${ }^{\circ} \mathrm{C}$, durante $72 \mathrm{~h}$. Realizaram-se as análises do tecido foliar seco seguindo-se metodologia descrita por Malavolta (1987) e após a coleta das plantas foi efetuada a amostragem do solo de cada vaso, coletando-se sub-amostras em quatro pontos de cada vaso $(0-20 \mathrm{~cm})$, com auxílio de uma sonda, cujo material foi então homogeneizado e submetido a análise química de fertilidade, através da metodologia descrita por Raij \& Quaggio (1983). Para determinação de teor de cromo total nas amostras de solo, utilizou-se $0,5 \mathrm{~g}$ de solo que foi digerido em de 6 $\mathrm{mL}$ de $\mathrm{HNO}_{3}$ concentrado, em duas parcelas de $3 \mathrm{~mL}$, conforme método de extração proposto por Missio (1996) com determinação em espectrofotômetro de absorção atômica.

O delineamento experimental adotado foi o de blocos inteiramente casualizado com sete tratamentos e quatro repetições e as médias comparadas pelo teste de Tukey a 5\%; o programa estatístico utilizado foi o Sisvar.

\section{RESULTADOS E DISCUSSÃO}

Os resultados encontrados na avaliação de altura de plantas e produção de massa seca demostraram resposta significativa do milho aos tratamentos que receberam lodo de curtume in natura (Tabela 2). Os tratamentos nos quais se adicionou este resíduo, proporcionaram ganhos de matéria seca significativamente maiores que os tratamentos testemunha e com adubação mineral em ambos os cultivos; resultados semelhantes foram encontrados por Konrad \& Castilhos (2002) que constataram produção de matéria quatro vezes a mais que a testemunha ao incorporarem lodo de curtume ao solo, para o cultivo do milho. Esses autores justificam, ainda, que os rendimentos observados com a aplicação do resíduo bruto são ratificados pela liberação imediata do $\mathrm{N}$ orgânico e subseqüente transformação em forma e teores suficientes para o desenvolvimento normal das plantas.

Os tratamentos que receberam o lodo de curtume compostado apresentaram valores de crescimento semelhantes aos

Tabela 2. Avaliação de crescimento no milho (doiscultivos sucessivos), em diversos tratamentos, aos 60 dias após o plantio

\begin{tabular}{|c|c|c|c|c|}
\hline \multirow{3}{*}{ Tratamento } & \multicolumn{2}{|c|}{ Primeiro cultivo } & \multicolumn{2}{|c|}{ Segundo cultivo } \\
\hline & Altura & $\begin{array}{c}\text { M. seca } p . \\
\text { aérea }\end{array}$ & Altura & $\begin{array}{l}\text { M. seca } p . \\
\text { aérea }\end{array}$ \\
\hline & $(\mathrm{cm})$ & (g) & $(\mathrm{cm})$ & (g) \\
\hline $1 T_{1}$ (Testemunha) & $67,3 d^{1}$ & $67,3 d^{1}$ & $52,0 \mathrm{~b}$ & $6,8 a$ \\
\hline $\mathrm{T}_{2}$ (NPK) & $99,5 b c$ & 99,5 bc & $56,0 \mathrm{~b}$ & $6,9 a$ \\
\hline$T_{3}$ (NK + fosforita) & $90,0 \mathrm{~cd}$ & $90,0 \mathrm{~cd}$ & $54,0 \mathrm{~b}$ & $6,6 \mathrm{a}$ \\
\hline$T_{4}$ (Lodo + fosforita) & $119,5 a b$ & $119,5 a b$ & $77,0 \mathrm{a}$ & $14,1 \mathrm{~b}$ \\
\hline $\mathrm{T}_{5}$ (Lodo (2x)+ fosforita) & $130,0 \mathrm{a}$ & $130,0 \mathrm{a}$ & $72,5 a b$ & $14,7 \mathrm{~b}$ \\
\hline $\mathrm{T}_{6}$ (Composto + fosforita) & $77,5 \mathrm{~cd}$ & $77,5 \mathrm{~cd}$ & $61,5 a b$ & $11,8 \mathrm{ab}$ \\
\hline $\mathrm{T}_{7}$ (Comp. $(2 \mathrm{x})+$ fosforita) & $76,2 \mathrm{~cd}$ & $76,2 \mathrm{~cd}$ & $55,5 \mathrm{~b}$ & $8,2 b$ \\
\hline
\end{tabular}

1-Médias seguidas de mesma letra não diferem entre si pelo teste de Tukey (5\%)

Tabela 3. Componentes de fertilidade do solo em diversos tratamentos, aos 60 dias após o plantio, em dois cultivos sucessivos

\begin{tabular}{|c|c|c|c|c|c|c|c|c|c|c|}
\hline \multirow{3}{*}{ Tratamento } & \multicolumn{5}{|c|}{ Primeiro cultivo } & \multicolumn{5}{|c|}{ Segundo cultivo } \\
\hline & $\mathrm{pH}$ & M.0. & $\mathbf{P}$ & K & $\mathrm{Ca}$ & $\mathrm{pH}$ & M.0. & $\mathbf{P}$ & $\mathbf{K}$ & $\mathrm{Ca}$ \\
\hline & $\left(\mathrm{CaCl}_{2}\right)$ & \multicolumn{2}{|c|}{$\left(g \mathrm{dm}^{-3}\right)$} & \multicolumn{2}{|c|}{$(\mathrm{mmol} \mathrm{dm}-3)$} & $\left(\mathrm{CaCl}_{2}\right)$ & \multicolumn{2}{|c|}{$\left(g_{d^{3}}^{3}\right)$} & \multicolumn{2}{|c|}{$\left(\mathrm{mmol} \mathrm{dm} \mathrm{m}^{-3}\right)$} \\
\hline $1 \mathrm{~T}_{1}$ (Testemunha) & 5,8 & 8,5 & $21,3 b^{1}$ & 1,06 & 11,3 & 5,7 & 11,0 & $13,5 d$ & 1,2 & 16,0 \\
\hline $\mathrm{T}_{2}$ (NPK) & 5,4 & 9,0 & $34,0 a b$ & 0,65 & 10,5 & 5,5 & 11,0 & $3,5 b c$ & 0,8 & 19,5 \\
\hline $\mathrm{T}_{3}$ (NK + fosforita) & 5,8 & 10,2 & $29,3 a b$ & 0,77 & 10,5 & 6,1 & 11,0 & $7,0 \mathrm{~cd}$ & 0,7 & 27,5 \\
\hline $\mathrm{T}_{4}$ (Lodo + fosforita) & 6,2 & 10,5 & $44,0 \mathrm{a}$ & 0,63 & 13,3 & 6,4 & 13,5 & $30,3 a b$ & 0,6 & 21,0 \\
\hline $\mathrm{T}_{5}$ (Lodo $(2 \mathrm{x})+$ fosforita) & 6,6 & 12,8 & 39,8 a & 0,63 & 31,5 & 7,2 & 16,0 & $38,5 \mathrm{a}$ & 0,4 & 47,0 \\
\hline $\mathrm{T}_{6}$ (Composto + fosforita) & 6,3 & 9,3 & $33,5 \mathrm{ab}$ & 0,93 & 16,5 & 6,2 & 12,0 & $19,5 \mathrm{~cd}$ & 0,7 & 20,5 \\
\hline $\mathrm{T}_{7}$ (Composto $(2 \mathrm{x})+$ fosforita) & 6,4 & 10,5 & $42,8 \mathrm{a}$ & 1,18 & 16,5 & 6,8 & 12,5 & $25,7 b c$ & 0,7 & 24,0 \\
\hline
\end{tabular}

1. Médias seguidas de mesma letra não diferem entre si pelo teste de Tukey (5\%) 
Tabela 4. Acúmulo de macronutrientes (mg por planta) do tecido foliar do milho após 60 dias de cultivo

\begin{tabular}{|c|c|c|c|c|c|c|c|c|c|c|}
\hline \multirow{2}{*}{ Tratamentos } & \multicolumn{5}{|c|}{ Primeiro cultivo } & \multicolumn{5}{|c|}{ Segundo cultivo } \\
\hline & $\mathbf{N}$ & $\mathbf{P}$ & K & $\mathrm{Ca}$ & $\mathbf{S}$ & $\mathbf{N}$ & $\mathbf{P}$ & K & $\mathrm{Ca}$ & $\mathbf{S}$ \\
\hline$\overline{1 \mathrm{~T}_{1} \text { (Testemunha) }}$ & $45,6 \mathrm{e}^{1}$ & $13,8 \mathrm{c}$ & $85,2 \mathrm{C}$ & $37,2 \mathrm{C}$ & $8,4 \mathrm{c}$ & $71,4 \mathrm{~b}$ & $17,6 \mathrm{~b}$ & $57,4 \mathrm{~b}$ & $21,6 \mathrm{~b}$ & $9,48 b$ \\
\hline $\mathrm{T}_{2}(\mathrm{NPK})$ & $211,4 \mathrm{c}$ & $31,2 b$ & $244,7 b$ & $113,5 b$ & $28,9 \mathrm{~b}$ & $84,5 b$ & $24,0 \mathrm{~b}$ & $70,1 a b$ & $26,1 b$ & $16,4 a b$ \\
\hline $\mathrm{T}_{3}$ (NK + fosforita) & $182,7 \mathrm{C}$ & $32,6 \mathrm{~b}$ & 332,8 a & $82,6 \mathrm{~b}$ & $28,3 \mathrm{~b}$ & $88,3 b$ & $18,4 \mathrm{~b}$ & $88,3 a b$ & $20,4 b$ & $10,5 \mathrm{~b}$ \\
\hline$T_{4}$ (Lodo + fosforita) & $322,4 \mathrm{~b}$ & 46,5 a & $368,9 a$ & 133,3 a & $49,6 \mathrm{c}$ & $197,1 \mathrm{a}$ & 36,6 a & $107,0 a b$ & $76,0 \mathrm{a}$ & $22,5 a b$ \\
\hline$T_{5}$ (Lodo $(2 x)+$ fosforita) & $424,7 a$ & 46,5 a & 409,2 a & 136,4 a & $49,6 \mathrm{c}$ & $214,4 a$ & $47,0 \mathrm{a}$ & $94,0 \mathrm{ab}$ & $70,5 \mathrm{a}$ & $26,4 a$ \\
\hline $\mathrm{T}_{6}$ (Composto + fosforita) & $78,3 \mathrm{ed}$ & $17,1 \mathrm{c}$ & $176,4 \mathrm{bc}$ & $37,8 \mathrm{c}$ & $10,8 \mathrm{c}$ & $165,1 \mathrm{a}$ & $36,5 a$ & 114,4 a & $37,7 \mathrm{ab}$ & $21,2 a b$ \\
\hline $\mathrm{T}_{7}$ (Composto $(2 \mathrm{x})+$ fosforita) & $111,1 \mathrm{~d}$ & $25,3 b c$ & $138,6 \mathrm{c}$ & 55,0 bc & $14,6 \mathrm{c}$ & $87,3 \mathrm{~b}$ & $28,0 \mathrm{ab}$ & $60,2 \mathrm{~b}$ & $28,8 \mathrm{~b}$ & $13,2 b$ \\
\hline
\end{tabular}

1 - Médias seguidas de mesma letra não diferem entre si pelo teste de Tukey (5\%)

da testemunha. A falta de resposta da cultura neste tratamento pode ser devido ao fato da maior estabilização do $\mathrm{N}$ no composto promover sua liberação mais lenta, o que pode ser comprovado pelo segundo cultivo do milho, em que apenas o tratamento com composto proporcionou ganhos no desenvolvimento da cultura quando se comparam os dois cultivos sucessivos; já se comprovou que a compostagem de um resíduo orgânico estabiliza o $\mathrm{N}$ que vai ser disponibilizado no solo a médio e longo prazo (Bar-Tal et al., 2004).

Com relação ao fornecimento de fósforo observou-se, na adubação do milho, pela análise de fertilidade do solo efetuada após a coleta das plantas, que a fosforita contribuiu significativamente para o aumento da quantidade de fósforo solúvel no solo, nos tratamentos associados ao lodo no segundo cultivo (Tabela 3), demonstrando, assim, que a matéria orgânica e a atividade microbiana no solo, incrementadas pelo resíduo orgânico, podem ter propiciado condições para a solubilização do $\mathrm{P}$ da fosforita e aumento na sua disponibilidade para as plantas. Konrad \& Castilhos (2001) comprovaram o aumento da atividade e população microbiana pela adição de lodo de curtume ao solo. Bactérias, fungos e actinomicetos estão envolvidos nos processos de solubilização e mineralização de P no solo, desempenhando papel fundamental no ciclo biogeoquímico desse elemento (Richardson, 2001). $\mathrm{O}$ efeito direto do lodo de curtume no aumento da disponibilização do fósforo foi confirmado pelos valores baixos de fósforo disponível no solo encontrado no tratamento com fosforita e adubação mineral; outro efeito nos tratamentos que receberam o lodo, diz respeito ao aumento no índice de $\mathrm{pH}$ e concentração de cálcio no solo, o que comprova o efeito corretivo do resíduo orgânico e confirma o que foi concluído por Ferreira et al. (2003) sobre o efeito de redução de acidez e aumento de cálcio trocável no solo que recebeu lodo de curtume.

A concentração de cromo no solo ficou abaixo de $1 \mathrm{ppm}$ em todos os tratamentos, valor este muito inferior ao valor de intervenção (300 ppm) indicado pela Legislação Ambiental Estadual (CETESB, 2001); Castilhos et al. (2002) constataram, em trabalho com aveia, que mesmo em tratamento com adição de cromo mineral não se notou translocação siginificativa deste metal para a parte aérea das plantas.

Verificou-se, com relação ao acúmulo de nutrientes pelo milho, que as plantas cultivadas em solo com lodo de curtume "in natura" e fosforita apresentaram aumentos significativos de nitrogênio e fósforo, nos dois cultivos, quando comparados com o tratamento apenas com a adubação convencional (Tabela 4) e, ainda, que o desenvolvimento das plantas no segundo cultivo foi inferior ao primeiro (Tabela 2) devido, em parte, à deficiência na absorção de potássio revelada pela análise foliar no segundo cultivo (Tabela 4), já previsto, em virtude dos baixos índices do elemento encontrado na análise de solo no primeiro cultivo (Tabela 3). O lodo de curtume é um resíduo que apresenta concentrações baixas de potássio (Ferreira et al., 2003).

Observa-se, também, que o tratamento que recebeu a menor dose de composto mais fosforita apresentou ganhos significativos no acúmulo de nitrogênio e fósforo pelas plantas, de um cultivo para o outro, Tabela 4, mostrando, daí, que este tratamento mineralizou o nitrogênio mais lentamente e aumentou a disponibilização do fósforo no segundo cultivo.

Com base nesses resultados, o lodo de curtume mostrou-se, nas quantidades avaliadas na adubação de plantio, promissor para fornecimento de nitrogênio ao cultivo do milho. Ferreira et al. (2003) afirmaram que a adição de $21 \mathrm{Mg} \mathrm{ha}^{-1}$ de lodo de esgoto na cultura do milho supriu a necessidade de $\mathrm{N}$ para o desenvolvimento da cultura, concluindo que até o estádio de florescimento as plantas apresentavam, na parte aérea, $47 \mathrm{~kg} \mathrm{ha}^{-1} \mathrm{de} \mathrm{N}$ a mais que o tratamento testemunha. A quantidade máxima de lodo de curtume utilizada neste trabalho $\left(5,0 \mathrm{Mg} \mathrm{ha}^{-1}\right)$ foi suficiente para proporcionar ganhos de desenvolvimento do milho. A análise de acúmulo de cromo no solo também revelou valores muito baixos; desta forma se afirma que a disposição de resíduo, utilizando-se lodo com baixo teor de cromo e nas quantidades aqui avaliadas, pode derivar em ganhos agrícolas com redução do impacto ambiental.

\section{CONCLUSÕES}

1. O lodo de curtume in natura nas doses aplicadas proporcionou desenvolvimento de milho superior ao obtido com a adubação mineral, com reduzido acúmulo de cromo no solo.

2. O lodo de curtume compostado proporcionou menor acúmulo de nutrientes no milho quando comparado com o lodo in natura, no primeiro cultivo.

3. O lodo de curtume associado à fosforita, proporcionou aumento do teor de fósforo solúvel no solo e maior absorção desse elemento pelo milho, nos dois cultivos.

4. A fosforita, associada ao resíduo, pode ser indicada como fonte de fornecimento de fósforo para o cultivo do milho. 


\section{LITERATURA CITADA}

Araujo, F. F.; Tiritan, C. S. Creste, J. E. Desenvolvimento da cultura do milho em solo tratado com lodo de curtume In: Congresso Brasileiro de Ciência do Solo, 30, 2005, Recife. Anais... Recife: UFRPE, 2005. CD Rom

Bar-Tal, A.; Yermiyahu, U.; Rosenberg, R.; Zohar, D. Rosen, V. Fine, P. Nitrogen, phosphorus and potassium uptake by wheat and their distribution in soil following sucessive, annual compost aplications. Journal Environmental Quality, v.33, n.5, p.1855-1865, 2004.

Branco, S. M.; Murgel, P. H.; Cavinatto, V.M. Compostagem: Solubilização biológica de rocha fosfática na produção de fertilizante organomineral. Engenharia Sanitária e Ambiental, v.6, n.3, p.115-122, 2001.

Castilhos, D. D. Alterações químicas e biológicas do solo resultantes da adição de resíduos de curtume e cromo hexavalente. Porto Alegre: UFRGS, 1998. 194p. Tese Doutorado

Castilhos, D. D.; Tedesco, M. J.; Vidor, C. Rendimentos de culturas e alterações químicas do solo tratado com resíduos de curtume e cromo hexavalente. Revista Brasileira de Ciência do Solo, v.26, n.5, p.1083-1092, 2002.

CETESB - Companhia de Tecnologia em Saneamento Ambiental. Relatório de estabelecimento de valores orientadores para solos e águas subterrâneas. São Paulo: CETESB, 2001. 73p.

EMBRAPA - Empresa Brasileira de Pesquisa Agropecuária. Sistema Brasileiro de Classificação de Solos. Rio de Janeiro: Embrapa Solos, 1999. 412p.

Ferreira, A. S.; Camargo, F. A. O.; Tedesco, M. J.; Bissani, C. A. Alterações de atributos químicos e biológicos de solo e rendimento de milho e soja pela utilização de resíduos de curtume e carbonífero. Revista Brasileira de Ciência do Solo, n.4, v.27, p.755-763, 2003.
Hussain, A. A.; Ghalia, H. H. A.; Abdallah, S. A. Rock phosphate solubilization by Aspergilli species on olive-cake waste and its application in plant growth improvement. Egyptian Journal of Biology, v.3, p.89-96, 2001.

Konrad, E. E.; Castilhos, D. D. Atividade microbiana em um planosolo após adição de resíduos de curtume. Revista Brasileira de Agrociência, v.7, n.2, p.131-135, 2001.

Konrad, E. E.; Castilhos, D. D. Alterações químicas do solo e crescimento do milho decorrentes da adição de lodos de curtume. Revista Brasileira de Ciência do Solo, v.26, n.1, p.257265, 2002.

Kucey, R.M.N.; Janzen, H.H.; Legget, M.E. Microbially mediated increases in plant-available phosphorus. Advances in Agronomy, v.42, p.199-228, 1989.

Lopes, A.S. Solos sob "cerrado": Características, propriedades e manejo. Piracicaba: Potafós, 1984. 162p.

Malavolta, E. Manual de química agrícola: Adubos e adubação. São Paulo: Livroceres, 1987. 596p.

Missio, E. Avaliação da disponibilidade de alguns metais pesados para as plantas. Porto Alegre: UFRGS, 1996. 120p. Dissertação Mestrado

Raij, B. van; Quaggio, J. A. Método de análise de solo para fins de fertilidade. Campinas: IAC, 1983. Boletim Técnico, 81

Richardson, A. E. Prospects for using soil microrganism to improve the acquisition of phosphorus by plants. Australian Journal of Plant Physiology, v.28, n.9, p.897906, 2001.

Tsunechiro, A.; Ferreira, C. R. P. T. Aspectos econômicos da adubação do milho no Brasil. Informações Econômicas, v.26, n.8, p.21-28, 1996. 